\title{
Metal-Insulator Transition in Copper Oxides Induced by Apex Displacements
}

\author{
Swagata Acharya, ${ }^{1, *}$ Cédric Weber, ${ }^{1, \dagger}$ Evgeny Plekhanov, ${ }^{1}$ Dimitar Pashov, ${ }^{1}$ A. Taraphder, ${ }^{2}$ and Mark Van Schilfgaarde ${ }^{1}$ \\ ${ }^{1}$ King's College London, Theory and Simulation of Condensed Matter, The Strand, \\ WC2R 2LS London, United Kingdom \\ ${ }^{2}$ Department of Physics and Centre for Theoretical Studies, Indian Institute of Technology \\ Kharagpur, India 721302
}

(Received 11 December 2017; revised manuscript received 24 March 2018; published 10 May 2018)

\begin{abstract}
High temperature superconductivity has been found in many kinds of compounds built from planes of $\mathrm{Cu}$ and $\mathrm{O}$, separated by spacer layers. Understanding why critical temperatures are so high has been the subject of numerous investigations and extensive controversy. To realize high temperature superconductivity, parent compounds are either hole doped, such as $\mathrm{La}_{2} \mathrm{CuO}_{4}$ (LCO) with $\mathrm{Sr}$ (LSCO), or electron doped, such as $\mathrm{Nd}_{2} \mathrm{CuO}_{4}(\mathrm{NCO})$ with $\mathrm{Ce}(\mathrm{NCCO})$. In the electron-doped cuprates, the antiferromagnetic phase is much more robust than the superconducting phase. However, it was recently found that the reduction of residual out-of-plane apical oxygen dramatically affects the phase diagram, driving those compounds to a superconducting phase. Here we use a recently developed first-principles method to explore how displacement of the apical oxygen (AO) in LCO affects the optical gap, spin and charge susceptibilities, and superconducting order parameter. By combining quasiparticle self-consistent GW (QS GW) and dynamical mean-field theory (DMFT), we show that LCO is a Mott insulator, but small displacements of the apical oxygen drive the compound to a metallic state through a localization-delocalization transition, with a concomitant maximum in $d$-wave order parameter at the transition. We address the question of whether NCO can be seen as the limit of LCO with large apical displacements, and we elucidate the deep physical reasons why the behavior of NCO is so different from the hole-doped materials. We shed new light on the recent correlation observed between $T_{c}$ and the charge transfer gap, while also providing a guide towards the design of optimized high- $T_{c}$ superconductors. Further, our results suggest that strong correlation, enough to induce a Mott gap, may not be a prerequisite for high- $T_{c}$ superconductivity.
\end{abstract}

Since their discovery, the electronic structure of high temperature superconductors [1-3] has been a subject of intensive theoretical attention, as well as controversy, a situation that continues even today. A landmark question to understand these materials is how their physical properties follow from their electronic and structural properties, and how key parameters govern the maximum critical temperature $T_{c}^{\max }$. Relating $T_{c}^{\max }$ [4] to a single variable has eluded physicists so far, because the observed correlation between $T_{c}^{\max }$ and structural properties across materials involves many kinds of them. Also, the recent finding of the impact of the reduction of residual out-of-plane apical oxygen on its phase diagram and superconductivity [5] triggers our theoretical experiment. In this work, we propose this novel

\footnotetext{
*swagata.acharya@kcl.ac.uk

†cedric.weber@kcl.ac.uk
}

Published by the American Physical Society under the terms of the Creative Commons Attribution 4.0 International license. Further distribution of this work must maintain attribution to the author(s) and the published article's title, journal citation, and DOI. systematic theoretical experiment, where we study the electronic modifications induced by displacements of the apical oxygen $(\mathrm{AO})$, in a typical copper oxide with the $\mathrm{T}$ perovskite structure. Recently, two main mechanisms have been put forward to explain the variation in $T_{c}^{\max }$ : (i) onebody terms involving out-of-plane molecular orbitals (axial orbitals with circular symmetry in the basal plane involving a mixture of $\mathrm{Cu} 4 \mathrm{~s}, \mathrm{Cu} 3 d_{z^{2}-3 r^{2}}$, apical oxygen $2 p_{z}$ ) [6], and (ii) many-body effects stemming from the in-plane Mott physics [4,7], either in one- or three-band models. In this work, we examine this picture carefully by using a state-ofthe-art approach, which treats both the many-body effects and one-electron terms on the same footing. We study the parent compounds $\mathrm{La}_{2} \mathrm{CuO}_{4}$ (LCO) of prototypical holedoped compounds $\mathrm{La}_{2-x} \mathrm{Sr}_{x} \mathrm{CuO}_{4}$ (LSCO). We use a realistic theoretical approach, namely, the quasiparticle self-consistent GW theory combined with dynamical mean-field theory [8,9] (QS GW + DMFT) [10], as implemented in the Questaal package [11] for QS GW, and Haule's implementation of DMFT [12] (see Appendix). GW and DMFT taken together allow for a better treatment of both nonlocal and local fluctuations. The parent pristine cuprate compound has for decades been known for its 
complexities in both local and nonlocal fluctuations. As we show, when the $\mathrm{AO}$ are displaced from their equilibrium position, the role of nonlocal fluctuations in spin, charge, and orbital sectors is nontrivially modified. The present scheme allows us to address many of these entangled issues in both single- and two-particle sectors. We carefully analyze how single-particle states, the bare charge transfer energy, the correlated spectral functions, and the optics evolve with displacement of the $\mathrm{AO}$ from its equilibrium position, without distorting the otherwise octahedral geometry and the crystal symmetry. We represent the displacement of the $\mathrm{AO}$ along the $c$ axis by $\delta$, such that the $(\mathrm{Cu}, \mathrm{AO})$ bond length expands or increases from its pristine geometry for $\delta>0$, and contracts for $\delta<0$. We evaluate the local irreducible vertex to construct the dynamical spin and charge susceptibilities, respectively, $\chi_{s}(Q, \Omega)$ and $\chi_{c}(Q, \Omega)$ [13].

Figure 1(a) shows the charge gap extracted from the spectral functions of LCO upon displacement $\delta$. Firstly, we find that pristine LCO is a paramagnetic Mott insulator, with a charge gap of approximately $0.7 \mathrm{eV}$. The imaginary part of the $\mathrm{Cu} d_{x^{2}-y^{2}}$ self-energy $\Sigma_{d}(i \omega)$ [see Fig. 1(c)] is singular with a large degree of incoherence.

For small, positive $\delta(<0.03 \AA)$, the system remains an insulator (MI) and we observe a direct correlation of the charge gap with the $\mathrm{Cu}-\mathrm{AO}$ distance. This can be explained by the reduction of the scattering rate [see Fig. 1(c)], which in turn reduces the incoherence and spread of the spectral weight features in the lower and upper Hubbard bands. This is also supported by the band structure provided in the Supplemental Material (SM) [14] (see Fig. 1 of the SM).

For $\delta>0.03 \AA$, the imaginary part of self-energy does not have a pole and is not Fermi-liquid-like; i.e., it is not linear in Matsubara frequencies. However, it has a finite (albeit extremely small) quasiparticle weight $Z$ [see Fig. 1(d)]. The incoherence is mostly lost, and we obtain, hence, a crossover from a MI to a charge transfer insulator (CTI). At critical displacement $\delta_{c}=0.045$, the gap collapses, and we obtain a correlated metal for $\delta>0.045 \AA$ (Fermi liquid).

We now turn to the discussion of the superconducting order parameter [see Fig. 1(a)]. We estimate the $d$-wave superconducting pairing correlator following the method outlined in Ref. [15] and discussed in more detail in the SI. We use the magnitude of the static d-wave order parameter as a proxy for the maximum superconducting temperature at optimal doping $\left(T_{c}^{\max }\right)$ [7]. Remarkably, we find that $T_{c}^{\max }$ [7] is largest at the localization-delocalization transition. This was already obtained in model Hamiltonian studies [16]. We confirm this model Hamiltonian prediction in a realistic framework: The apex displacement provides more than a gedanken experiment; it provides a realization of the Mott transition in a given material with a nearly $a b$ initio Hamiltonian, by varying a single control parameter. Our analysis indicates that the phase transition at $\delta_{c}$ shapes not only the normal-state phase diagram, but strikingly leaves its mark on the complex structure of the superconducting
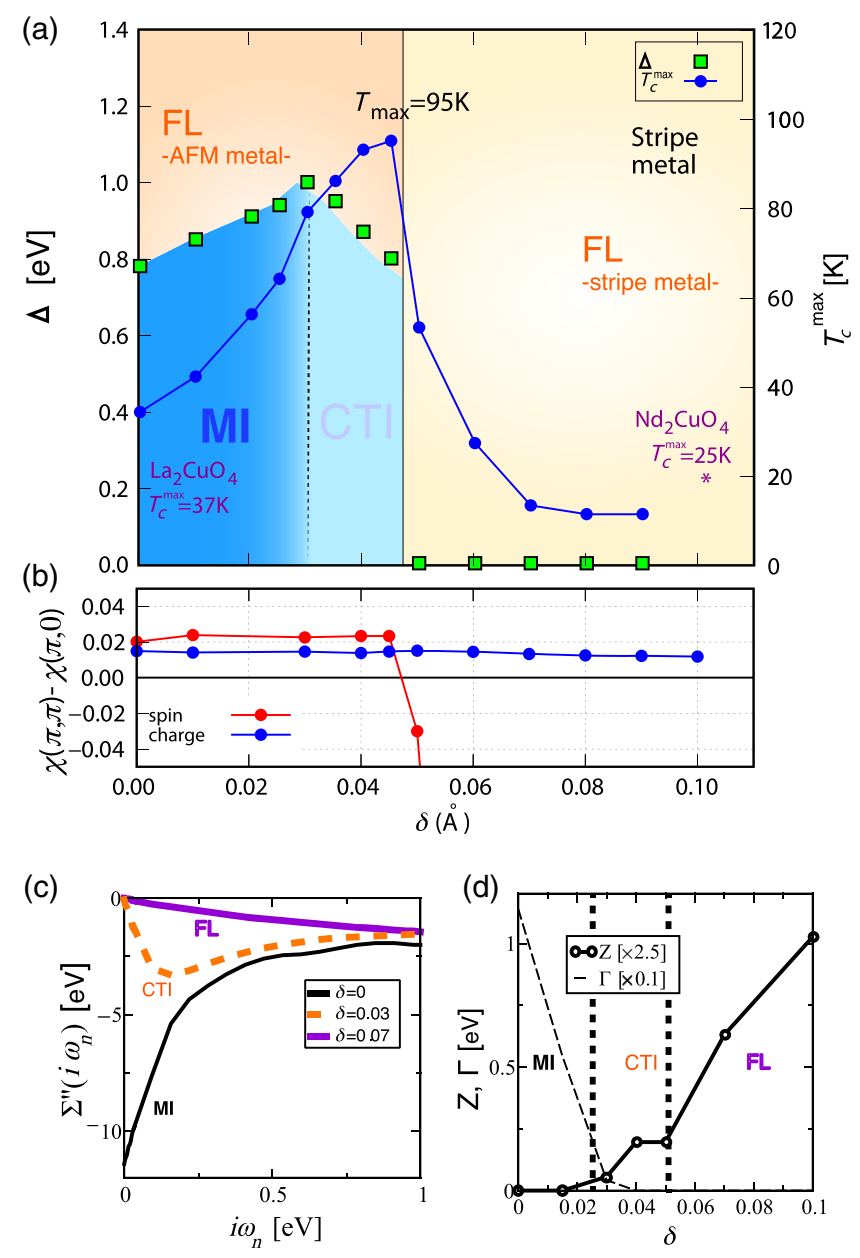

FIG. 1. Phase diagram. (a) Charge gap $\Delta$ (squares, left ordinates) with respect to $\delta$, the apical oxygen displacement. We show also the estimated critical temperature at optimal doping $T_{c}^{\max }$ (circles, right ordinates). We find that optimum critical temperature is largest at the insulator to metal transition $(\delta=0.045 \AA)$. (b) Relative static susceptibilities $\chi(\pi, \pi)-\chi(\pi, 0)$ for spin (blue circles) and charge (red circles) degrees of freedom. (c) Imaginary part of the $\mathrm{Cu} d_{x^{2}-y^{2}}$ self-energy $\Sigma^{\prime \prime}(i \omega)$ on the Matsubara axis for pristine LCO (bold line) and distorted LCO. For $\omega<0.03$, the self-energy is extrapolated with a polynomial fit to $\omega=0$. The large scattering rate and the pole in the self-energy for $\delta<0.02$ indicate a Mott insulating phase (MI), whereas for $\delta>0.03 \AA$, we obtain a charge transfer insulator (CTI) with nonquadratic self-energies. (d) Imaginary part of the self-energy extrapolated to zero frequency $\Gamma=$ $-\Sigma^{\prime \prime}(i \omega \rightarrow 0)$ and quasiparticle weight $\mathrm{Z}$.

condensate that is born out of this unusual normal state. It provides, hence, a guide towards the design of optimized superconductors. Correlating the $T_{c}^{\max }$ with a single parameter for the cuprates seemed difficult in those earlier studies, as different chemical compositions have several different parameters. However, our calculations allow us to achieve this ideal experiment, where a single structural parameter is changed, and we can measure its effect on the electronic properties. This provides key insights into identifying what mechanisms control the electronic structure. 
Furthermore, an antilinear correlation between $T_{c}^{\max }$ and the charge gap has been suggested both on theoretical [7] and experimental grounds [4]. We note that a similar trend is obtained in our data for the CTI phase: The charge gap is reduced as the apex distance is shifted by more than $\delta=0.03 \AA$, but the critical temperature $T_{c}^{\max }$ increases up to the maximum of approximately $100 \mathrm{~K}$ at $\delta_{c}$. This is understood in the picture of a loss of correlation strength, which in turn increases $T_{c}^{\max }$ in the vicinity of the delocalization transition, a many-body origin.

Interestingly, for small but positive $\delta(<0.03 \AA)$ the increase of $T_{c}^{\max }$ correlates with the $\mathrm{Cu}-\mathrm{AO}$ distance. This matches with the early prediction of Pavarini et al. [6]. They concluded that $T_{c}^{\max }$ is controlled by the energy of the axial orbital, a hybrid between $\mathrm{Cu} 4 \mathrm{~s}, \mathrm{AO} 2 p_{z}$, and $\mathrm{Cu} 3 \mathrm{~d}$ orbitals. Our results provide a similar trend with the $\mathrm{Cu}-\mathrm{AO}$ distance for $\delta<0.03 \AA$. Indeed, for pristine LCO and for small displacements $(\delta<0.03 \AA)$, the QS GW band structure indicates that the lower Hubbard d band of pristine LCO, a hybrid between Cu- $d_{x^{2}-y^{2}}$ and in-plane oxygens, also has approximately $10 \%$ axial character [see Fig. 1(a) and the table of the Supplemental Material [14]]. The formation of the axial orbital stems from the one-body part of the Hamiltonian, and it is present in both DFT and GW; however, the nonlocal fluctuations in GW suppress the axial orbital contribution at the Fermi level significantly.

Finally, we note that the phase diagram is extremely asymmetric in $\delta$. We obtain insulating DMFT solutions for compressions of the AO up to $\delta=-0.05 \AA$, and the charge gap increases monotonically for all such displacements. This is explained by an increased charge-transfer energy scale for $\mathrm{Cu}-d_{x^{2}-y^{2}}$ and $\mathrm{O}-p_{x, y}$.

Remarkably, at the critical displacement $\delta_{c}$, we observe a concomitant change of momentum in the spin fluctuations associated with the gap collapse. We show in Fig. 2(a) the momentum resolved static magnetic susceptibility for pristine LCO. The susceptibility is peaked at $\mathbf{Q}=(\pi, \pi)$, but with a broad width, denoting short-range antiferromagnetic superexchange $J$ in direct space. For displacements larger than $\delta_{c}$, we observe a clear shift of the peak to $\mathbf{Q}=(\pi, 0)$ [Fig. 2(c)].

The relative static susceptibilities $\chi(\pi, \pi)-\chi(\pi, 0)$ provide an order parameter to track the change of magnetic pitch vector [see Fig. 1(b)]. While the spin fluctuations are located at $\mathbf{Q}=(\pi, \pi)$ for pristine LCO and $\delta<\delta_{c}$, they abruptly evolve to $\mathbf{Q}=(\pi, 0)$ after the transition [see Fig. 1(b)].

This is reminiscent of the finite asymmetry present in the orthorhombic structure of pristine LCO [17]. Since the orthorhombic phase does not respect the $x-y$ rotational invariance, the peak is larger at $(0, \pi)$ than $(\pi, 0)$. As the incoherence is lost, and the quasiparticle weight increases, the spectral features near the gap edge recover the small structural asymmetry (for small Z, the spectral features in the lower band are flat), and in turn provides a magnetic

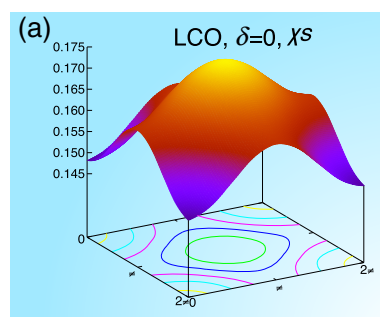

(c)
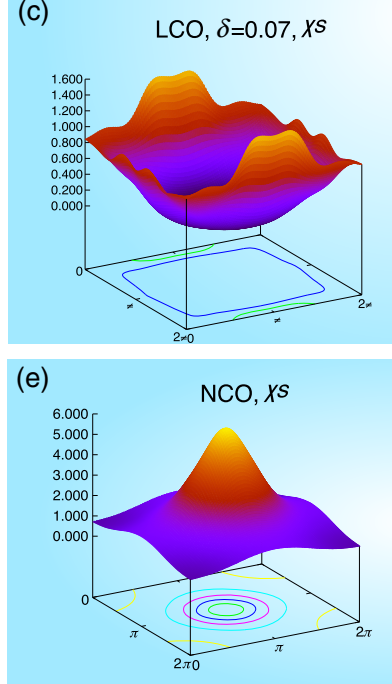

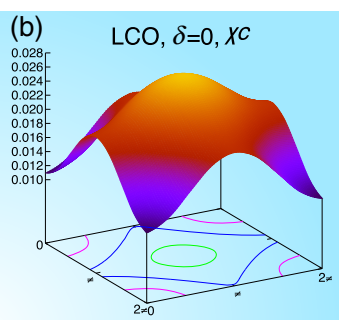

(d)
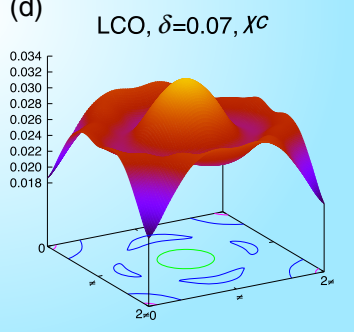

FIG. 2. Spin and charge fluctuations: static spin fluctuations $X^{s}(k, \omega=0)$ for (a) pristine LCO, (c) distorted LCO $(\delta=0.07 \AA)$, and (e) $\mathrm{Nd}_{2} \mathrm{CuO}_{4}(\mathrm{NCO})$. Static charge fluctuations $X^{c}(k, \omega=0)$ for (b) pristine LCO, (d) distorted LCO ( $\delta=0.07 \AA$ ), and (f) NCO.

transition from the antiferromagnetic to a striped metallic phase. Interestingly, the charge fluctuations remain peaked for LCO at all times at $\mathbf{Q}=(\pi, \pi)$ [see Figs. 2(b) and 2(d)], and they remain small in amplitude throughout the transition.

Under compression $(\delta<0)$, the spin susceptibility peak survives at $Q=(\pi, \pi)$, suggesting that there is no significant change in the nesting and AFM fluctuations.

Once the $\mathrm{AO}$ is pushed far from $\mathrm{Cu}$ (large $\delta$ ), a localization-delocalization transition with a concomitant magnetic transition from AFM to a stripe phase, as is evidenced by the change in the shape and strength of the spin susceptibility. The latter drives charge fluctuations, which reduce the local moment and the gap.

To derive further insight into the microscopic mechanism driving this transition, we analyze the evolution of the energy levels and their orbital characters across the transition. The pioneering work of Zaanen, Sawatzky, and Allen (ZSA) [18] and their sharp (so-called ZSA) boundary between charge transfer metals and charge transfer insulators is the canonical picture to describe the copper oxides. The variation of the charge transfer gap [see Fig. 1(a)] suggests naively that the charge transfer energy between the in-plane $\mathrm{Cu} 3 d$ and the in-plane oxygens is affected, as it is believed to determine the strength of correlations in a charge transfer system. 
It would be expected that the $\mathrm{Cu}-\mathrm{AO}$ distance would directly affect the in-plane charge transfer energy: The negatively charged apical oxygen increases the electrostatic potential at the copper site, and as it is removed we expect a decrease of the charge transfer energy, $\Delta_{x}=\epsilon_{p_{x}}-\epsilon_{d_{x^{2}-y^{2}}}$.

However, our calculations show that the electrostatic charge transfer energy changes only by $0.2 \mathrm{eV}$ across the transition (see Fig. 3). This effect is, however, sufficient to drive the compound to a metal and, hence, confirms that LCO is sitting at the boundary between a metal and insulator.

We also find that the out-of-plane $\mathrm{Cu}-\mathrm{AO}$ charge transfer is changing on an energy scale of approximately $0.1 \mathrm{eV}$ (see Fig. 3). The latter stems from the rehybridization between the $\mathrm{Cu} 3 d$ and out-of-plane orbitals, as surmised by Pavarini et al. [6], via nonlocal correlation effects captured by the GW. Indeed, we observe that the band at the Fermi level has approximately $20 \%$ character from the axial orbitals $\left(d_{z^{2}}, \mathrm{O}-p_{z}, \mathrm{Cu}-4 \mathrm{~s}\right)$ within the LDA calculations. The GW reduces this weight down to approximately $10 \%$, but we observe that this remains present for
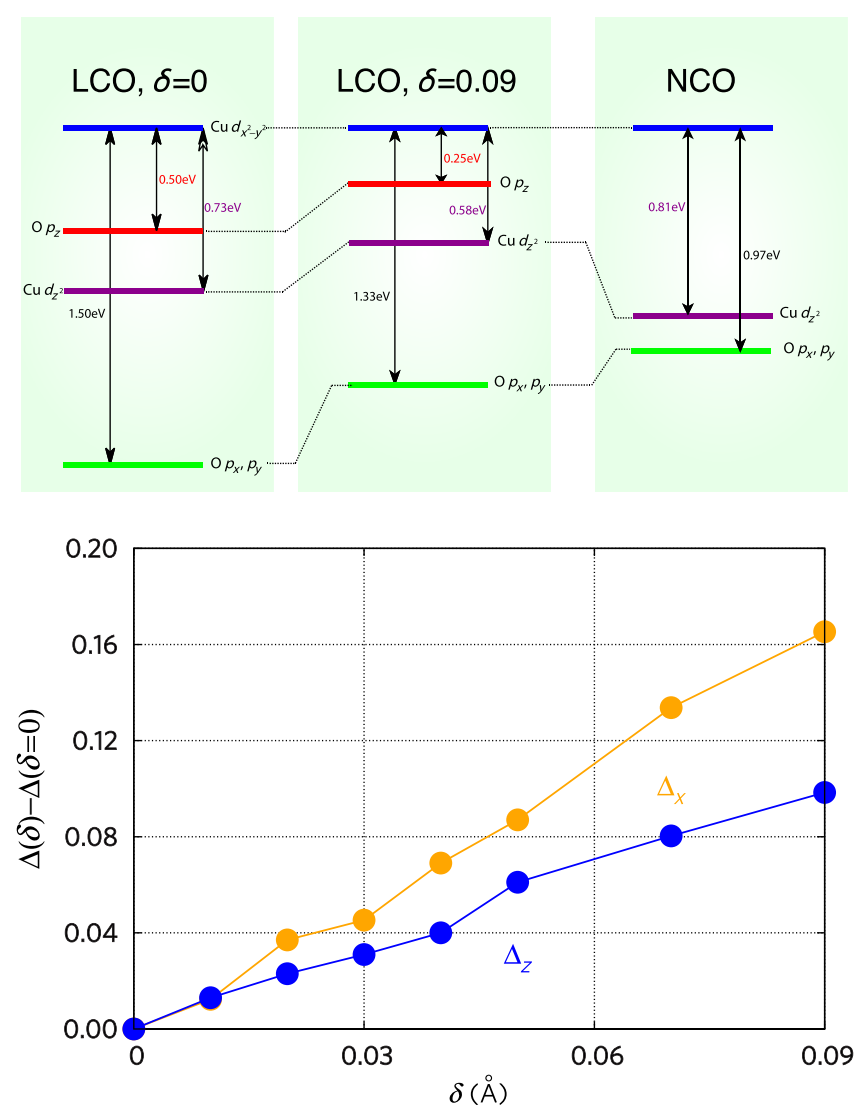

FIG. 3. Energy levels and charge transfer energy. Upper panel: The relative positions of the relevant QS GW orbitals for $\delta=0,0.09 \AA$ and NCO. Lower panel: Variation upon apex displacement of the in-plane charge transfer energy $\Delta_{x}=$ $\epsilon_{p_{x}}-\epsilon_{d_{x^{2}-y^{2}}}$ and of the out-of-plane $\mathrm{Cu}$-AO charge transfer energy $\Delta_{z}=\epsilon_{p_{z}}-\epsilon_{d_{3 z^{2}-r^{2}}}$. the largest AO displacements. Interestingly, the axial character is nearly suppressed in NCO due to the absence of $\mathrm{AO}$, with $d_{z^{2}}, \mathrm{O}-p_{z}$, not contributing at all and $\mathrm{Cu}-4 s$ contributing approximately 3\%. This suggests that the physics in NCO is well described by a three-band model. We also find that NCO has a significantly reduced $\Delta_{x}=$ $0.97 \mathrm{eV}$ (see Fig. 3) than LCO $\left(\Delta_{x}=1.50 \mathrm{eV}\right)$.

We now turn to the optical conductivity $\sigma(\omega)$ (see Fig. 4). A good description of the optical conductivity depends on a good description of single-particle states. We find that while the DFT $\sigma(\omega)$ is far from the experimental data, $\sigma(\omega)$ calculated from QS GW alone, without local correlations provided by DMFT, gives rise to a fairly good description, apart from a Drude term at low frequency. This is a consequence of QS GW's ability to generate a oneparticle Hamiltonian with a good description of quasiparticle levels in many kinds of materials systems. Some explanation of the differences between QS GW and DFT are given in the Supplemental Material [14]. However, QS GW cannot create a Mott gap. The local self-energy from DMFT Mott gaps the system and kills the Drude peak. When QS GW and DMFT are combined, we find an optical gap of approximately $1.8 \mathrm{eV}$ with a sharp onset at around $2.0 \mathrm{eV}$, with $\sigma=920(\mathrm{ohm}-\mathrm{cm})^{-1}$, followed by a plateau extending to nearly $3.5 \mathrm{eV}$, in remarkable agreement with the data extracted from Ref. [19]. This provides a strong validation of the theory for pristine LCO, which is very important: Previous theoretical attempts had difficulty in obtaining a good description of the onset and the plateau, and further corrections were invoked. A DMFT calculation using a six-band model, including the apical oxygen and $d_{z^{2}-3 r^{2}}$ orbitals, is not able to resolve the discrepancy between theoretical optics and the experiments [20].

The QS GW self-energy, with its spatial nonlocality and nonlocality in time through dynamical screening, has been shown to yield consistently good QP levels, and this seems to be the case in LCO as well. In particular, it aligns the $\mathrm{O}-p$ and the $\mathrm{Cu}-d$ states very differently than DFT, and places

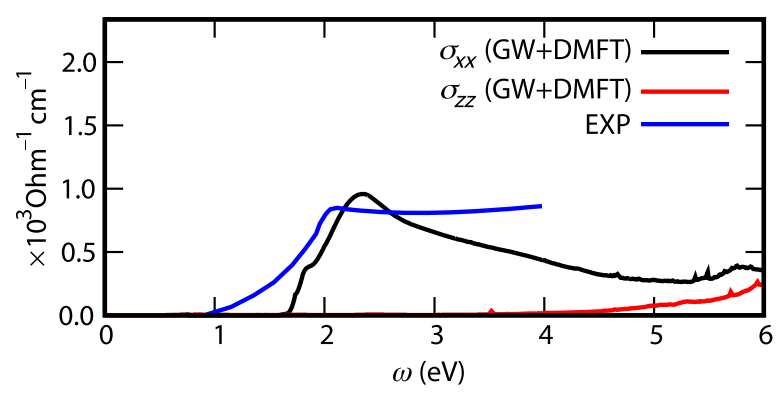

FIG. 4. Optics: theoretical optical conductivity $\sigma(\omega)$ obtained with the QS GW + DMFT approach for the in-plane $\left(\sigma_{x x}\right)$ and out-of-plane components $\left(\sigma_{z z}\right)$. We emphasize that these results are a prediction from an essentially ab initio Hamiltonian. We obtain remarkable agreement with experimental data, reproduced from Ref. [19], which validates the theoretical approach. 
La $f$ states far above $E_{F}$. (See also the SM [14].) There remain some discrepancies with experiment: The shoulder and height of the peak are well described, but less so the plateau above the shoulder. Also, the spectral weight for $\Omega>6 \mathrm{eV}$, taken from later experiments but not published in Ref. [19], is blueshifted. Both of these effects are to be expected because of the missing vertex in the random phase approximation (RPA) [21], a well-known deficiency of the RPA. DMFT corrections are negligible for $\Omega>3 \mathrm{eV}$.

Performing a similar calculation for NCO, we find it to be a correlated metal, with a single orbital active at the Fermi level, an admixture of $d_{x^{2}-y^{2}}$ and in-plane oxygen (see Table I in the Supplemental Material [14]). One might expect that its metallic nature would share similarities with the limiting case of large AO displacements for LCO. We find, however, that, despite the apparent similarities in the one-particle response functions, the two metallic phases are qualitatively very different, as shown by their two-particle responses. For instance, we find very different magnetic [see Fig. 2(e)] and charge [see Fig. 2(f)] susceptibilities. Firstly, the charge fluctuations are peaked at the $\Gamma$ point, in stark contrast with LCO, where the charge fluctuations are peaked at $(\pi, \pi)$. Second, the background of charge fluctuations in NCO is two orders of magnitude larger than for LCO. This is, indeed, consistent with the recent experimental predictions for $\mathrm{NCO}$ that found a metallic ground state under protected annealing [22-24]. Finally, the magnetic susceptibility is peaked at $(\pi, \pi)$, but the peak is relatively narrow, suggesting long-range antiferromagnetic superexchange interactions in direct space.

The strong spin and charge separation in NCO susceptibilities suggests that the dynamics can no longer be described in terms of individual particles or single-particle excitations, as pointed out earlier by Anderson [25]. Although it is not definite proof, our results indicate that $\mathrm{NCO}$ is likely to go through a superconducting instability, rather than through a spin- or charge-density wave alone. Our results agree, hence, with the recent observation that annealed $\mathrm{NCO}$ and $\mathrm{Pr}_{2} \mathrm{CuO}_{4}$ (PCO) compounds are superconducting, and not antiferromagnetic [5].

\section{ACKNOWLEDGMENTS}

We thank Yayu Wang for discussions, insights, and experimental data and Lorenzo Sponza and Paolo Pisanti for help with the codes. This work was supported by EPSRC CCP9 (EP/M011631/1 and also EP/M011038/1) and the Simons Many-Electron Collaboration. C. W. gratefully acknowledges the support of the NVIDIA Corporation with the donation of the Tesla K40 GPUs used for this research. For computational resources, we were supported by the ARCHER UK National Supercomputing Service and the UK Materials and Molecular Modelling Hub for computational resources (EPSRC Grant No. EP/ P020194/1).

\section{APPENDIX: METHOD}

We use a recently developed quasiparticle self-consistent $\mathrm{GW}+$ dynamical mean-field theory (QS GW + DMFT) approach to address this problem, as implemented in the Questaal package [11]. There exist GW + DMFT [26-28] studies for materials and model Hamiltonians in the literature, but the approach is different from our recently developed QS GW + DMFT. Paramagnetic DMFT is combined with nonmagnetic QS GW via local projectors of the $\mathrm{Cu} 3 d$ states on the $\mathrm{Cu}$ augmentation spheres to form the correlated subspace. We carried out the QS GW calculations in the orthorhombic phase of LCO with space group $64 / \mathrm{Cmca}$ [17]. DMFT provides a nonperturbative treatment of the local spin fluctuations. We use an exact hybridization expansion solver, namely, the continuous time Monte Carlo (CTQMC) [12], to solve the Anderson impurity problem.

The one-body part of QS GW is performed on a $16 \times$ $16 \times 16 \mathrm{k}$-mesh and charge has been converged up to $10^{-6}$ accuracy, while the (relatively smooth) static self-energy $\Sigma^{0}(\mathbf{k})$ is constructed on a $4 \times 4 \times 4 \mathrm{k}$-mesh from the dynamical GW $\Sigma(\mathbf{k}, \omega) . \Sigma^{0}(\mathbf{k})$ is iterated until convergence [root mean square (RMS) change in $\Sigma^{0}<10^{-5}$ Ry]. $U=$ $10 \mathrm{eV}$ and $J=0.7 \mathrm{eV}$ [29] were used as correlation parameters for DMFT. The DMFT for the dynamical self-energy is iterated and converges in approximately ten iterations. The calculations for the single-particle response functions are performed with $10^{8}$ QMC steps per core and the statistics is averaged over 64 cores. The two-particle Green's functions are sampled over a larger number of cores (192) to improve the statistical error bars. We sample the local two-particle Green's functions with CTQMC for all the correlated orbitals and compute the local polarization bubble to solve the inverse BetheSalpeter equation (BSE) for the local irreducible vertex. Finally, we compute the nonlocal polarization bubble $G(\mathbf{k}, \omega) G(\mathbf{k}-\mathbf{Q}, \omega-\Omega)$ and, combined with the local irreducible vertex [13], we obtain the full nonlocal spin and charge susceptibilities $\chi_{s, c}(\mathbf{Q}, \Omega)$. Since here we are focused on the lowest energy excitations, we computed the momentum resolved, multiorbital, static $(\Omega=0)$ spin and charge susceptibilities. The susceptibilities are computed on a $16 \times 16 \times 16 \mathbf{Q}$-mesh.

[1] N. P. Armitage, D. H. Lu, C. Kim, A. Damascelli, K. M. Shen, F. Ronning, D. L. Feng, P. Bogdanov, X. J. Zhou, W. L. Yang, Z. Hussain, P. K. Mang, N. Kaneko, M. Greven, Y. Onose, Y. Taguchi, Y. Tokura, and Z.-X. Shen, AngleResolved Photoemission Spectral Function Analysis of the Electron-Doped Cuprate $\mathrm{Nd}_{1.85} \mathrm{Ce}_{0.15} \mathrm{CuO}_{4}$, Phys. Rev. B 68, 064517 (2003).

[2] Y. Tokura, A. Fujimori, H. Matsubara, H. Watabe, H. Takagi, S. Uchida, M. Sakai, H. Ikeda, S. Okuda, and S. Tanaka, Electron and Hole Doping in Nd-Based Cuprates 
with Single-Layer $\mathrm{CuO}_{2}$ Sheets: Role of Doped Ce Ions and 30-K Superconductivity, Phys. Rev. B 39, 9704 (1989).

[3] H. Takagi, S. Uchida, and Y. Tokura, Superconductivity Produced by Electron Doping in $\mathrm{CuO}_{2}$-Layered Compounds, Phys. Rev. Lett. 62, 1197 (1989).

[4] P. Cai, W. Ruan, Y. Peng, C. Ye, X. Li, Z. Hao, X. Zhou, D.-H. Lee, and Y. Wang, Visualizing the Evolution from the Mott Insulator to a Charge-Ordered Insulator in Lightly Doped Cuprates, Nat. Phys. 12, 1047 (2016).

[5] M. Horio, T. Adachi, Y. Mori, A. Takahashi, T. Yoshida, H. Suzuki, L. C. C. Ambolode II, K. Okazaki, K. Ono, H. Kumigashira et al., Suppression of the Antiferromagnetic Pseudogap in the Electron-Doped High-Temperature Superconductor by Protect Annealing, Nat. Commun. 7, 10567 (2016).

[6] E. Pavarini, I. Dasgupta, T. Saha-Dasgupta, O. Jepsen, and O. K. Andersen, Band-Structure Trend in Hole-Doped Cuprates and Correlation with Tc Max, Phys. Rev. Lett. 87, 047003 (2001).

[7] C. Weber, C. Yee, K. Haule, and G. Kotliar, Scaling of the Transition Temperature of Hole-Doped Cuprate Superconductors with the Charge-Transfer Energy, Europhys. Lett. 100, 37001 (2012).

[8] A. Georges, G. Kotliar, W. Krauth, and M. J. Rozenberg, Dynamical Mean-Field Theory of Strongly Correlated Fermion Systems and the Limit of Infinite Dimensions, Rev. Mod. Phys. 68, 13 (1996).

[9] K. Held, O. K. Andersen, M. Feldbacher, A. Yamasaki, and Y. F. Yang, Bandstructure Meets Many-Body Theory: The LDA + DMFT Method, J. Phys. Condens. Matter 20, 064202 (2008).

[10] L. Sponza, P. Pisanti, A. Vishina, D. Pashov, C. Weber, M. van Schilfgaarde, S. Acharya, J. Vidal, and G. Kotliar, SelfEnergies in Itinerant Magnets: A Focus on Fe and Ni, Phys. Rev. B 95, 041112 (2017).

[11] The quasiparticle electronic structure in augmented LMTOS (Questaal) code is available at https://www.questaal.org.

[12] K. Haule, Quantum Monte Carlo Impurity Solver for Cluster Dynamical Mean-Field Theory and Electronic Structure Calculations with Adjustable Cluster Base, Phys. Rev. B 75, 155113 (2007).

[13] H. Park, K. Haule, and G. Kotliar, Magnetic Excitation Spectra in $\mathrm{BaFe}_{2} \mathrm{As}_{2}$ : A Two-Particle Approach within a Combination of the Density Functional Theory and the Dynamical Mean-Field Theory Method, Phys. Rev. Lett. 107, 137007 (2011).

[14] See Supplemental Material at http://link.aps.org/ supplemental/10.1103/PhysRevX.8.021038 for details of QSGW self energy, comparative discussion for DFT and QSGW spectral functions, QSGW + DMFT spectral functions, importance of local and non-local correlations, computation of $T_{c}$ and a table summarizing all the parameters we computed from our theory for the relevant systems.

[15] A.-M. Daré, L. Chen, and A.-M.S. Tremblay, Comparisons between Monte Carlo Simulations and a Simple
Crossing-Symmetric Approach to the Hubbard Model at Low Density, Phys. Rev. B 49, 4106 (1994).

[16] L. Fratino, P. Sémon, G. Sordi, and A.-M. S. Tremblay, An Organizing Principle for Two-Dimensional Strongly Correlated Superconductivity, Scientific reports 6, 22715 (2016).

[17] M. Reehuis, C. Ulrich, K. Prokeš, A. Gozar, G. Blumberg, S. Komiya, Y. Ando, P. Pattison, and B. Keimer, Crystal Structure and High-Field Magnetism of $\mathrm{La}_{2} \mathrm{CuO}_{4}$, Phys. Rev. B 73, 144513 (2006).

[18] J. Zaanen, G. A. Sawatzky, and J. W. Allen, Band Gaps and Electronic Structure of Transition-Metal Compounds, Phys. Rev. Lett. 55, 418 (1985).

[19] S. Uchida, T. Ido, H. Takagi, T. Arima, Y. Tokura, and S. Tajima, Optical Spectra of $\mathrm{La}_{2-x} \mathrm{Sr}_{x} \mathrm{CuO}_{4}$ : Effect of Carrier Doping on the Electronic Structure of the $\mathrm{CuO}_{2}$ Plane, Phys. Rev. B 43, 7942 (1991).

[20] X. Wang, H. T. Dang, and A. J. Millis, $d_{3 z^{2}-r^{2}}$ Orbital in High- $T_{c}$ Cuprates: Excitonic Spectrum, Metal-Insulator Phase Diagram, Optical Conductivity, and Orbital Character of Doped Holes, Phys. Rev. B 84, 014530 (2011).

[21] S. Albrecht, L. Reining, R. Del Sole, and G. Onida, Ab Initio Calculation of Excitonic Effects in the Optical Spectra of Semiconductors, Phys. Rev. Lett. 80, 4510 (1998).

[22] H. J. Kang, P. Dai, B. J. Campbell, P. J. Chupas, S. Rosenkranz, P. L. Lee, Q. Huang, S. Li, S. Komiya, and Y. Ando, Microscopic Annealing Process and Its Impact on Superconductivity in T-Structure Electron-Doped Copper Oxides, Nat. Mater. 6, 224 (2007).

[23] M. Ikeda, T. Yoshida, A. Fujimori, M. Kubota, K. Ono, Y. Kaga, T. Sasagawa, and H. Takagi, Effects of Annealing on the Electronic Structure of the Electron-Doped High-Tc Superconductor $\mathrm{Nd}_{1.85} \mathrm{Ce}_{0.15} \mathrm{CuO}_{4}$, J. Phys. Conf. Ser. 108, 012016 (2008).

[24] N. Hiasa, T. Saiki, and R. Ikeda, Vortex Lattice Structure Dependent on Pairing Symmetry in Rashba Superconductors, Phys. Rev. B 80, 014501 (2009).

[25] P. W. Anderson, Spin-Charge Separation Is the Key to the High Tc Cuprates, Physica (Amsterdam) 341-348C, 9 (2000).

[26] J. M. Tomczak, P. Liu, A. Toschi, G. Kresse, and K. Held, Merging GW with DMFT and Non-local Correlations Beyond, Eur. Phys. J. Spec. Top. 226, 2565 (2017).

[27] J. M. Tomczak, M. Casula, T. Miyake, F. Aryasetiawan, and S. Biermann, Combined GW and Dynamical Mean-Field Theory: Dynamical Screening Effects in Transition Metal Oxides, Europhys. Lett. 100, 67001 (2012).

[28] T. Ayral, S. Biermann, and P. Werner, Screening and Nonlocal Correlations in the Extended Hubbard Model from Self-Consistent Combined GW and Dynamical Mean Field Theory, Phys. Rev. B 87, 125149 (2013).

[29] S. Choi, A. Kutepov, K. Haule, M. van Schilfgaarde, and G. Kotliar, First-Principles Treatment of Mott Insulators: Linearized QS GW + DMFT Approach, npj Quantum Mater. 1, 16001 (2016). 